\title{
Welchen Sinn es hat, vom «Vertrauen» in Gott zu reden?
}

Welchen Sinn hat es, vom «Vertrauen» in Gott zu reden? Diese Frage stellt sich weniger dem Atheisten, mehr schon dem skeptisch Sinnsuchenden und interessanterer Weise gerade einem gläubigen Christen. Kann die Beziehung zum Gott Jesu Christi angemessen durch «Vertrauen» umschrieben bzw. muss sie neuzeitlich gar dadurch ersetzt werden oder ist der einzig angemessene Ausdruck dieser Bezugnahme auf Gott mit «Glauben» erreicht? Und falls für Letzteres optiert wird: verweist das «Vertrauen» in Gott nicht zumindest auf einen für den Glaubensvollzug wesentlichen Aspekt? Das jedenfalls würde die in derTheologiegeschichte exponierte Stellung der fiducia stützen, bei der insbesondere seit ihrer reformatorischen Betonung durch Luther und Melanchthon, in systematisierter Weise dann seit der lutherischen Orthodoxie, die fides in assensus, notitia und fiducia explizit auf den Glaubensvollzug bezogen wurde.

Um die Frage nach dem Sinn der Rede vom «Gottesvertrauen» beantworten zu können, will ich zunächst auf das Phänomen des zwischenmenschlichen Vertrauens fokussieren, um im Anschluss daran abwägen zu können, ob aufgrund charakteristischer Eigenschaften ihres sozialen Vollzuges Vertrauen auch geeignet ist, die menschliche Ausrichtung auf Gott metaphorisch zu beschreiben oder als Vertrauen $\mathrm{zu}$ vollziehen.

\section{Vertrauen als Phänomen zwischenmenschlicher Erfahrung}

Vertrauen ist ein Phänomen menschlicher Erfahrung. Das zu konstatieren unterlässt kaum ein Beitrag zu Vertrauen. ObwohlVertrauen - trotz empirisch noch so ausgeklügelten Versuchsanlagen - aus der Aussenperspektive nicht recht $\mathrm{zu}$ fassen ist, wird keiner bestreiten, dass ein Mensch innerhalb seines Lebens nicht in irgendeiner Weise, irgendwann, irgendetwas oder irgendwem vertraut. Daran möchte auch dieser Artikel festhalten, und das sicher nicht nur, um damit das Objekt eigener Forschungsbemühungen zu rechtfertigen. Ich gehe vielmehr davon aus, dass sich menschliches Leben in einem Gefüge 
von Situationen vollzieht, in denen Vertrauen zu einer Möglichkeit wird, die nicht ohne Grund immer wieder wirksam realisiert wird.

Heiss umstrittene Fragen nach dem Ursprung des Vertrauens (liegt er in einer anthropologischen Fähigkeit der Person, im ¿Zwischen〉 eines Beziehungsgeschehens, im Einbezug in eine bestehende Vertrauenspraxis) und der Teilnahmeart des Vertrauenden (affektive Haltung, Verhaltensdisposition, den Personen äusserlich zukommendes Geschehen) können dabei aussen vor gelassen werden.Vielmehr will ich allein den Charakter des Vertrauens aufspüren und anhand dessen abzuwägen versuchen, ob inhärente Eigenschaften von Vertrauensvollzügen zur Beschreibung des Glaubensvollzuges in signifikanter Weise dienen oder nicht.

Hilfreich für diese Aufgabenstellung wird sein,Vertrauen zunächst innerhalb seiner vielfältigen Vollzugsformen aufzuspüren. Denn für Vertrauen gilt, dass es nicht nur die eine Form des Vertrauens gibt, sondern dass Vertrauen in unterschiedlichen Situationen und Horizonten, in unterschiedlichen Bezügen, mit unterschiedlichen Objekten, in unterschiedlicher Intensität und Bedingtheit gelebt wird. Diese Pluriformität gilt es erst einmal wahrzunehmen und theoretisch anzuerkennen, um sie im Anschluss für den systematischen Umgang greifbar zu machen.

Der Begriff des «Vertrauens» - das wird einem bei einiger Aufmerksamkeit darauf schnell klar - fällt in der Alltagssprache allzu häufig. Als Forscherin, der es um Klarheit und Präzision dieses sinnlich nicht fassbaren Phänomens gelegen ist, stellt einen dieser Befund zunächst vor erkenntnistheoretische Probleme; und dann vor methodische Entscheidungen: Die Vielfältigkeit an Verwendungen für den Begriff des Vertrauens soll hier anhand von Wittgensteins Modell der 〈Familienähnlichkeiten` gehandhabt werden. Ich gehe also davon aus, dass es unterschiedliche aber doch miteinander verwandte Vertrauensformen, Vertrauenssituationen, Vertrauensbeziehungen gibt, die Menschen in mehr oder weniger grosser (Un)Schärfe mit «Vertrauen» zum Ausdruck bringen. Weder soll mithilfe einer abstrakten Definition ein Richtmass für die Sprachverwendung gesetzt, noch sollen Vertrauensbekundungen der Willkür überlassen werden. Anhand ihrer semantischen Ausdrucksgestalten möchte ich im Folgenden einzelne zwischenmenschliche Vertrauensvollzüge sichtbar machen, um im Anschluss daran eine Ordnung ihrer Charakteristika zu erstellen. 


\section{«Ich vertraue dir»}

«Ich vertraue dir!» ist jene Aussage, die wohl als Grundform aller Vertrauensäusserungen verstanden werden kann. Sie ist Ausdruck einesVertrauens zwischen Person und Person. Ich gehe davon aus, dass diese personale Konstellation jene (individualgeschichtlich) grundlegende Vertrauenserfahrung darstellt, die im Laufe der lebenslangen Vertrauenspraxis weiter ausdifferenziert, vielfältig entfaltet oder auch funktionalisiert wird. Sie bildet zudem die sprachliche Ausgangsbasis, d.h. dient als Art Modellsatz für Vertrauenserfahrungen, die auch differenzierter zu formulieren möglich wären:

\section{«Ich vertraue dir etwas an»}

«Ich vertraue dir meinen Kanarienvogel über die Sommerferien an» steht für eine solche sekundäre Vertrauensform, die sich von der Grundform des personalen Vertrauens ableitet und begrifflich klarer als anvertrauen unterscheidbar ist. Beim Anvertrauen handelt es sich um eine dreistellige Relation, bei der das vertrauende Subjekt einem Gegenüber ein (grammatisches) Objekt anvertraut, jenem in die Obhut gibt und für eine gewisse Zeit überlässt. Das Vertrauen bezieht sich hierbei darauf, dass die Bezugsperson innerhalb eines gegebenen Spielraumes nach den Erwartungen des Vertrauenden handelt und mit dem anvertrauten Objekt angemessen umgeht.

\section{«Ich verlasse mich auf dich hinsichtlich...» ${ }^{1}$}

Dem Anvertrauen kommt das Sich-in-einer-Hinsicht-Verlassen sehr nahe. Es entspricht sich darin, dass eine vertrauende Person auf eine andere Person im Hinblick auf etwas Drittes baut. Allerdings unterscheidet es sich darin, dass die vertrauende Person sich selbst (in einer bestimmten Hinsicht) auf die andere Person verlässt. D.h. es geht nicht um das Abgeben eines dritten Objektes in die Obhut des anderen, sondern darum, sich selbst einem anderen (in seinem Wünschen, Bedürfnissen etc.) auszusetzen. «Ich verlasse mich auf dich hinsichtlich unseres gemeinsamen Klettervorhabens», wäre ein Beispiel für diese Vertrauensform.

1 Das "sich-verlassen-auf» verwende ich hier als dem "vertrauen» derVerwandtschaft nach sachlich äquivalente Formulierung. Mit «sich-verlassen-auf» können bestimmte Felder des Vertrauens spezifischer angezeigt werden; jene nämlich, bei denen die vertrauende Person selbst (in existenziellen ebenso wie in bestimmten Hinsichten) auf dem Spiel steht. 


\section{«Ich verlasse mich auf etwas»}

Sich auf etwas zu verlassen hat offensichtlich die Differenz zum vorhergehenden Sich-Verlassen darin, dass die vertrauende Person sich nicht auf jemanden verlässt, sondern auf etwas. D.h. hier vertraut der Vertrauende nicht in ein personales Gegenüber, sondern in ein technisches Gerät, System oder eine Institution. «Ich verlasse mich auf den Airbus A380» fällt ebenso unter diese Kategorie wie «ich verlasse mich auf die ZKB». In beiden Fällen vertraut eine Person auf die Verlässlichkeit dessen, was das Flugzeug qua technischem Gerät bzw. die Bank qua Institution verspricht.

«lch verlasse mich existenziell auf...»

Sich schliesslich existenziell auf jemanden oder etwas $\mathrm{zu}$ verlassen ist die radikalste Art zu vertrauen. Bei einem existenziellen Vertrauensvollzug verlässt sich eine Person mit ihrem ganzen Leben (und Sterben) auf jemanden, dem sie die Erfüllung dieser umfassenden Erwartung zutraut. Sich jemandem existenziell ganz anzuvertrauen gipfelt in der Hoffnung, dass letztlich «alles gut wird». Paradigmatisch vertrauen sich Gläubige in dieser Weise ihrem Gott an - in Not und Freude, im Leben wie im Sterben. Funktional äquivalent verhält es sich mit jenen Formen des Vertrauens, die in der Forschung als «Seinsvertrauen» oder «Grundvertrauen» beschrieben werden. Diese Begriffen wollen ähnlich existenzielle Vertrauensvollzüge beschreiben - allerdings explizit ohne Bezug auf ein göttliches Gegenüber.Vielmehr wird dabei in die Stabilität, Tragfähigkeit und Güte des Lebenszusammenhangs in der Welt vertraut, darauf, dass es 〈das Leben〉 gut mit einem meint.

Im Folgenden gehe ich dazu über, in all diesen Situationen und Weisen zu vertrauen, Ähnlichkeiten bzw. Verwandtschaften aufzusuchen. Zunächst möchte ich Vertrauen dafür als Bezugs- bzw. Beziehungsphänomen kennzeichnen. Denn so verschiedenartig die Vertrauensformen auch sind, in jeder gibt es mindestens zwei Pole, die miteinander in ein Verhältnis treten. Vertrauen findet entweder zwischen zwei (oder mehreren) Menschen statt oder - was aber als sekundäre Form gegenüber interpersonalen Beziehungen zu werten ist - jemand verlässt sich auf eine Institution, ein technisches Gerät oder System. So oder so gibt es Bezüge zwischen den am Vertrauensgeschehen Einbezogenen, ohne die Vertrauen niemals zustande käme.

Zudem erstrecken sich Vertrauensphänomene in den Verlauf der Zeit. Vertrauen ist ein transtemporales Phänomen. Ein 
Vertrauensvollzug ist dementsprechend nicht eigentlich erfasst, wenn etwa auf eine Momentaufnahme vom Gehirn eines vertrauenden Menschen verwiesen wird.Vollständig erfasst werden kann Vertrauen erst in seiner Erstreckung auf Bewährung oder Enttäuschung. Vertrauensäusserungen reichen immer aus einer erfahrungsbezogenen, mit rationalen, emotionalen oder intuitiven Beweggründen gestützten Gegenwart in die Zukunft hinein.

Eine weitere, wesentliche Eigenschaft des Vertrauens wird daraus ersichtlich: ihr Wagnischarakter. Unter einem Wagnis verstehe ich hier jede mit nicht kalkulierbaren Ungewissheiten behaftete, mit Hoffnung auf einen positiven Ausgang aber ergriffene Vorleistung, bei der die eigene Person auf dem Spiel steht. Zwar wird Vertrauen längst nicht in allen Fällen als bewusst gegen Ungewissheiten entschiedene Handlung vollzogen. Dennoch kann jeder auch un-, vor- oder unterbewusst vollzogene Vertrauensakt entweder im Nachhinein oder aber von einem Dritten als «Wagnis» beschrieben werden. Der Wagnischarakter ist deshalb so zentral, weil im Vertrauen eine Verhaltensmöglichkeit ergriffen wird, die sich dadurch auszeichnet, gerade ohne gesuchte (obwohl zu suchen mögliche) Absicherung und Kontrolle zu sein. "Wer nicht wagt, der nicht vertraut» wäre insofern ein Motto, welches zum Ausdruck bringt, dass manche wertvollen Lebens- und Beziehungsdimensionen erst dadurch zu entstehen die Möglichkeit haben, wenn unkalkuliert zu vertrauen gewagt wird.

Den Mut, Vertrauen zu wagen, kann deshalb aufgebracht werden, weilVertrauen von einer positiven Erwartungshaltung durchsetzt ist. Im Vertrauen wird Gutes erwartet. Man spricht nicht von «Vertrauen», wenn Übles erwartet oder Negatives prophezeit wird. Positiv sind Erwartungen beim Vertrauen insofern, als darauf vertraut wird, dass die andere es schlicht "gut mit einem» meint; dass das anvertraute Objekt im Sinne desVertrauenden und dem anvertrauten Gegenstand entsprechend gut gehandhabt wird; oder schliesslich dass technische Geräte, Institutionen und Systeme halten, was man zurecht von ihnen erwartet. Das im Gegensatz zu Kontrolle und Sicherstellung Vertrauensspezifische daran ist, dass demVertrauenden die konkreten Schritte des Anderen zur Erfüllung der Vertrauenserwartung verborgen sind. Dem ins Vertrauen Gezogenen sind Ermessensspielräume überlassen, von denen derVertrauende ausgeht, dass sie wohlwollend und zum Guten gebraucht werden.

Schliesslich gilt es für alle Vertrauensformen anzunehmen, dass ihr Vertrauen auf Evfahrung und gemeinschaftlich gepflegter Vertrauens-Kultur basiert. Das heisst: zum Vertrauen wird befähigt und ermutigt, wem 
selbst schon einmal Vertrauen entgegen gebracht wurde oder wem sich Vertrauen als gangbare Lebensmöglichkeit erwiesen hat. Ich gehe insofern davon aus, dass Vertrauen nicht schon als anthropologische Eigenschaft oder angeborene Anlage in jedem Säugling steckt (etwa als sog. «Urvertrauen»), sondern dass es eine gemeinschaftliche Vertrauenspraxis voraussetzt, in der sich einem Menschen diese Lebensmöglichkeit eröffnet.

Soziale Vollzüge, die wir «Vertrauen» nennen, zeichnen sich zusammengefasst also dadurch aus, dass sie (personale) Beziehungen abbilden, sich über gewisse Zeiträume erstrecken, Wagnis in sich tragen, dass sie von positiven Erwartungen geprägt sind und auf Erfahrungen einer miteinbezogenen Vertrauenspraxis beruhen. Gibt es hinsichtlich dieser verwandtschaftlichen Eigenheiten nun $\mathrm{Ge}-$ meinsamkeiten mit jenem «Vertrauen», das in Gott gelegt wird?

\section{Gottesvertrauen - eine Metapher?}

Vertrauen - in all seinen Variationen und Horizonten - macht einen Unterschied für unser Dasein als Menschen. Und das nicht nur für den gemeinschaftlichen Lebensalltag, sondern auch für die religiöse Dimension unseres Lebens. Denn mit dem Vollzug des «Sich-selberverlassens-auf» kann qua Erfahrung eingelöst werden, was von Gott verheissen und religiös zugesprochen wird. Vertrauen könnte in spiritueller Betrachtung insofern als Erfahrungsbild für Gottes Verheissung und die religiöse Erwartung gelesen werden, erlöst von Vereinzelung und Selbstsorge in befreiter Gemeinschaft zu leben.

Wenden wir uns aber der umgekehrten und eigentlichen Frage nach der Beschreibung des Glaubensvollzuges als Vertrauen zu: Liefert die Erfahrung zwischenmenschlichen Vertrauens ein besonders geeignetes Sprachbild, eine Metapher für den Glaubensvollzug? Ist also Glaube wieVertrauen oder vollzieht sich Glaube als Vertrauen?

Nicht nur reformatorische (und gegenreformatorische) Akzentuierungen, nicht erst die lutherische Orthodoxie, sondern schon Thomas von Aquin und mittelalterliche Mystiker (Meister Eckhart, Johannes Tauler) verwendeten - ebenso wie Autoren der neueren Theologiegeschichte - den Begriff des Vertrauens im Zusammenhang von Glaubenslehren. Zu vermuten ist daher, dass all diese Theologen nicht ohne Grund zu diesem Begriff gegriffen haben. Zwei ausgewählte Beiträge möchte ich nun vorstellen und ihre theologischen Pointen in derVerwendung des Vertrauensbegriffs auf die eingangs ermittelten Vertrauenscharakteristika beziehen. 
Wilhelm Herrmanns Modell des Glaubens als Verkehr des Christen mit Gott sei zunächst vorgestellt, weil er in seinem so betitelten Werk von 1868, in dem er sich intensiv mit dem Wesen des Glaubens auseinandergesetzt und diesen als «Vorgang reinen Vertrauens» analysiert hat, zwei auch für die zwischenmenschliche Vertrauenserfahrung charakteristischen Akzente exponiert: Zentral ist Hermann zunächst die Beziehungsrelation im christlichen Glauben, bei dem «Gott selbst mit uns in Verkehr tritt» - «so wie damals die Jünger». Christlicher Glaube also beruht darauf, dass Gott auf uns zugeht, mit uns in ein Verhältnis tritt, woraus im Glauben eine Vertrauensbeziehung entsteht - wie das auch in zwischenmenschlichen Begegnungen der Fall sein kann. Ebenso wichtig erachtet Herrmann zweitens den Erfahrungsbezug des Glaubens. Die Erfahrung, die hier sehr bedeutend für den Glauben wird, richtet sich spezifisch auf das Selbsterleben der «Tatsache» Jesu. Der methodisch entscheidende Punkt in Herrmanns Erfahrungstheologie ist, dass der «objektive Grund", d.h. Gewissheit über die Sache Gottes, dem Christen allein durch dieses «selbsterlebte Ereignis» zukommen kann. Das Vertrauen auf die erlebbare Nähe Jesu, seine wirkungsvolle Kraft stellt daher den relevanten Schritt für die Glaubenskonstitution dar. «Niemand kann durch Beweise dazu gebracht werden. (...) Aber der Mensch, in dem das Vertrauen zu der Person Jesu erwacht ist, entbehrt nicht des objektiven Grundes für diese Haltung.» - Womit zugleich der Wagnischarakter des Vertrauens in seiner Überwindung angespielt ist.

Wolfhart Pannenberg theologische Verwendung des Vertrauensbegriffs bringt weitere Charakteristika des gläubigen Vertrauens zum Vorschein. Grundlegend für Pannenbergs «Vertrauensglaube» ist die Annahme, dass der Mensch (ähnlich wie in der Bezugnahme auf eine andere Person) über den göttlichen Ursprung der Gesamtwirklichkeit nicht verfügen kann. «Der Ursprung, in dem wir die Einheit alles Wirklichen suchen, um der Einheit unseres Daseins gewiss zu werden, kann darum, weil er unendlich ist, nur imVertrauen ergriffen werden.» Vertrauen steht also für einen Modus, in dem Unverfügbarem ebenso wie Unendlichem begegnet werden kann. Der Wagnischarakter in dieser Bezugnahme ist offensichtlich:Vertrauen steht für ein das Wagnis - aufgrund des faktischen Nichtwissens und Nichtverfügens - positiv in sich aufnehmendes, dem Leben und der Wahrheit angemessene «Sichverhalten», das dem neuzeitlichen Versuch der «Sicherstellung» gegenübergestellt wird. Die andere Charakteristik von Vertrauen, die sich im «Vertrauensglauben» Pannenbergs findet, ist ihre Zeitdurchwirktheit. Vertrauen in Gott hegt Pannenberg zufolge jener Glaube, der sich auf Gottes Verheissung einlässt und ihre zukünftige 
Erfüllung erwartet. Das Leben eines Gläubigen zeichnet sich dadurch aus, dass es nicht in Selbstsorge und Angst verharrt, sondern die unvorhersehbare Zukunft in vertrauensvoller Offenheit ergreift. Gerade das gibt ihm seine eschatologische Ausrichtung, und seine zeitliche Distanz zur Erfüllung begründet den Unterschied zum Schauen (2 Kor 5,7). Schliesslich sieht Pannenberg den Vertrauensglauben auch auf die Erfahrung bezogen. Obwohl Pannenberg zufolge dem Menschen (einer idea innata gleich) Vertrauen als Bezogenheit-worauf? in die Wiege gelegt ist, erhält sie ihre notwendige Konkretion über die Erfahrungen der Lebenswirklichkeit. «So oder so lebt jeder Mensch, so lange er lebt, aus einem sein Leben tragenden Grundvertrauen (...) Der Fortgang seines Lebens wird die Tragfähigkeit seines Vertrauens erweisen oder zeigen, dass er auf Sand gebaut hat.» Zwischenmenschliche Vertrauensbeziehungen erachtet Panneberg als vorläufige Manifestationen des eigentlich zu lebenden Gottesvertrauens. Das herauszufinden bedarf es allerdings des Umweges über zwischenmenschliche Vertrauenserfahrungen, angefangen mit jenem Urvertrauensverhältnis des Säuglings zur Mutter.

Der Glaube an den Gott Jesu Christi wird in diesen beiden ausgewählten Beiträgen seinem Vollzug und bei Herrmann auch der Konstitution nach mithilfe des Begriffes «Vertrauen» umschrieben. Dabei wurden mit der Beziehungsrelation zu Jesus Christus, der zeitlichen Erstreckung auf die eschatologische Erfüllung von Verheissungen ebenso wie mit der Erfahrungsgrundlage ihres Wagnisses einige jener Charakteristika angesprochen, die wir auch in zwischenmenschlichen Vertrauensvollzügen gefunden haben. ${ }^{2}$

Ist damit nun geklärt, ob Gottesglaube wie Vertrauen oder als Vertrauen vollzogen wird? So speziell die Grundlage der Wagnismotivation und so ausserordentlich die Person Gottes als Gegenüber auch sind - die Glaubensrelation beinhaltet Vollzüge, die unserem Verständnis von Vertrauen tatsächlich entsprechen. Nicht nur wie im zwischenmenschlichen Vertrauen lasse ich mich auf das existenzielle Wagnis von Gottes Verheissungen ein, sondern ich vertraue darauf. Nicht nur wie im Vertrauen beziehe ich mich auf Gott, sondern vertrauensvoll tue ich dies auf der Grundlage meiner Erfahrung und biblischer Zeugnisse. Vertrauen ist somit nicht lediglich Sprachbild

\footnotetext{
2 Für die analysierte positive Erwartungshaltung im Vertrauen könnte Martin Luther angeführt werden. In seiner berühmten Auslegung des 1. Gebots im Grossen Katechismus liest man im Zusammenhang der «Etymologie» des deutschen Wortes "Gott» von "gut» folgendes: «Der Sinn des Gebots: Dass man Gott alleine trauen und sich eitel Guts zu ihm versehen und von ihm gewarten soll [ist] (...) also, dass Gott alleine der ist, von dem man alles Guts empfängt und alles Unglücks los wird.» (BSLK 565f)
} 
oder Metapher für ein nicht anders analytisch greifbares Phänomen, sondern Gottesglaube wird menschlich (unter anderem) als Vertrauen vollzogen.

Dennoch unterscheidet sich Gottesvertrauen von anderen Vertrauensvollzügen nicht allein durch das (ausserhalb des Sakraments nicht leiblich anwesende) Gegenüber des Vertrauens. Obwohl ich tatsächlich in menschlicher Weise auch auf Gott vertraue, ist mein Gottesvertrauen in einen Kontext eingebettet, der dieses Vertrauen von anderen zwischenmenschlichen Vertrauensvollzügen spezifisch unterscheidet. Es ist der religiöse Horizont des Glaubens, vor dem das Gottesvertrauen seine Bestimmtheit erhält. Denn das Vertrauen in Gott steht niemals allein, sondern ist bezogen auf den Glauben an Gott und umgeben von anderen Glaubensvollzügen des Nachdenkens, des gemeinsamen Feierns und Betens. Ohne diese anderen Aspekte der Glaubenspraxis, die allesamt Gottes Selbsterweis und Glaubenskonstitution voraussetzen, bleibt jeder Vergleich mit zwischenmenschlichen Vertrauensvollzügen unzulänglich.

Dennoch: unsere menschliche Bezugnahme auf Gott mit dem uns wohl vertrauten Begriff des «Vertrauens» zu bezeichnen, macht Sinn. Es macht Sinn, eben weil zentrale Charaktereigenschaften von zwischenmenschlichen Vertrauensvollzügen auch im Glauben an Gott zum Tragen kommen. Dass das Gottesvertrauen zugleich eine kategorial andere Form von Vertrauen darstellt, weil es Teil eines Lebensvollzuges im Glauben ist, der von Gott begründet und alles auf Gott hin bezogen sein lässt, tut dem keinen Abbruch.

\section{Welchen Sinn es hat}

Während Bultmann die «Sinnfrage` bekanntlich hinsichtlich der «Rede von Gott» überhaupt problematisiert hat, steht es mit der ¿Sinnfrage` im Bezug auf die Anwendung des Vertrauensbegriffs auf den Glaubensvollzug anders. Dieser Beitrag hat sich weder die Rede von Gott noch die Rede von der menschlichen Existenz, sondern die Rede von einem Aspekt unseres Glaubensvollzuges vorgenommen. So wenig auch wir über den hiesigen Gegenstand, das Gottesvertrauen als Erfahrungswirklichkeit verfügen können, so sehr bin ich doch der Meinung, dass die Frage nach dem Sinn ihrer Rede legitim und notwendig zu stellen ist. Denn wer einmal in das Beziehungsgeschehen des Gottesvertrauens im Glauben miteinbezogen worden ist, der will - bzw. «muss» - darüber reden. 
Sicherlich wäre es notwendig, weitere theologische Dokumente auf ihre Pointen für die Verwendung des Vertrauensbegriffs zu befragen, um ein umfassenderes Bild für die gestellte Frage zu bekommen. Immerhin aber wurden einige wenige Parameter vorgestellt, die auf das im Glauben vollzogene Vertrauen hinweisen und damit nahe legen, welchen Sinn es tatsächlich macht, vom «Vertrauen» in Gott zu reden.

- Andrea Lassak arbeitet im Rahmen des Projekts «Vertrauen verstehen» an einer Dissertation mit dem Thema «Vertrauen im Horizont christlichen Glaubens». 